\title{
Curculigoside promotes osteogenic differentiation of ADSCs to prevent ovariectomized-induced osteoporosis
}

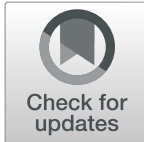

Wei-Li You ${ }^{1 *}$ and Zheng-Long $\mathrm{Xu}^{2}$

\begin{abstract}
Background: Curculigoside is a natural phenolic glycoside compound produced by Curculigo orchioides Gaertn. This study aimed to explore the effects of curculigoside in promoting the osteogenic differentiation of adiposederived stem cells (ADSCS) as well as the underlying mechanism.

Methods: ADSCs were treated with curculigoside at different concentrations $(0 \mu \mathrm{mol} / \mathrm{L}, 1 \mu \mathrm{mol} / \mathrm{L}, 2.5 \mu \mathrm{mol} / \mathrm{L}, 5$ $\mu \mathrm{mol} / \mathrm{L}, 10 \mu \mathrm{mol} / \mathrm{L}$, and $20 \mu \mathrm{mol} / \mathrm{L})$, and cell viability was assessed by CCK-8 assay. Then, the alkaline phosphatase (ALP) activity was determined, and alizarin red S (ARS) staining was performed to measure the extracellular mineralization of curculigoside. Information about protein-chemical interactions is provided by the search tool for interactions of chemicals (STITCH) database. Then, LY294002 was administered to explore the mechanism by which curculigoside promotes the osteogenic differentiation of ADSCs. Western blot assays were performed to assess changes in the expression of osteogenic-related markers and the phosphorylation of PI3K and AKT. Finally, we established an ovariectomized (OVX)-induced osteoporosis mouse model and administered curculigoside to explore the effects of curculigoside in preventing bone loss in vivo.

Results: The CCK-8 assay indicated that curculigoside did not induce cytotoxicity at a concentration of $5 \mu \mathrm{mol} / \mathrm{L}$ after $48 \mathrm{~h}$. The ALP and ARS results revealed that the induced group had higher ALP activity and calcium deposition than the control group. Moreover, the curculigoside group exhibited increased biomineralization, ALP activity, and ARS staining compared to the induced and control groups, and these effects were partially inhibited by LY294002. Kyoto Encyclopedia of Genes and Genomes (KEGG) enrichment analysis indicated that the target genes of curculigoside were mainly involved in the PI3K-Akt signaling pathway. PCR and western blot analysis showed that the expression of RUNX2, ALP, and Osterix was upregulated in curculigoside-treated ADSCs, but this effect was partially reversed by the PI3K inhibitor LY294002. Moreover, the curculigoside-treated group exhibited significantly increased phosphorylation of AKT to P-AKT compared with the osteogenic induction group. After treatment with curculigoside, the mice had a higher bone volume than the OVX mice, suggesting partial protection from cancellous bone loss. In addition, when LY294002 was added, the protective effects of curculigoside could be neutralized.

(Continued on next page)
\end{abstract}

\footnotetext{
* Correspondence: 16826000@163.com

'Department of Pharmacy, The First People's Hospital of Lianyungang, No.

128, Tongguanbei Road, Haizhou District, Lianyungang 222002, Jiangsu

Province, China

Full list of author information is available at the end of the article
}

\section{$\triangle B M C$}

(c) The Author(s). 2021 Open Access This article is licensed under a Creative Commons Attribution 4.0 International License, which permits use, sharing, adaptation, distribution and reproduction in any medium or format, as long as you give appropriate credit to the original author(s) and the source, provide a link to the Creative Commons licence, and indicate if changes were made. The images or other third party material in this article are included in the article's Creative Commons licence, unless indicated otherwise in a credit line to the material. If material is not included in the article's Creative Commons licence and your intended use is not permitted by statutory regulation or exceeds the permitted use, you will need to obtain permission directly from the copyright holder. To view a copy of this licence, visit http://creativecommons.org/licenses/by/4.0/ The Creative Commons Public Domain Dedication waiver (http://creativecommons.org/publicdomain/zero/1.0/) applies to the data made available in this article, unless otherwise stated in a credit line to the data. 
(Continued from previous page)

Conclusions: Curculigoside could induce the osteogenic differentiation of ADSCs and prevent bone loss in an OVX model through the PI3K/Akt signaling pathway.

Keywords: Adipose-derived stem cells, Curculigoside, PI3K/Akt signaling pathway, Osteoporosis

\section{Background}

Osteoporosis is a systemic bone metabolism disease that is caused by bone loss and bone microstructure damage [1-3]. As the population ages, the prevalence of osteoporosis increases year by year [4]. The pathogenesis of osteoporosis involves the disruption of the dynamic balance between bone resorption and bone formation [57]. Bone resorption exceeds bone formation, ultimately leading to bone mass decreases. Bone strength in osteoporosis patients is decreased, and the bones are prone to fracture [8]. Therefore, seeking suitable drugs to promote osteoblastic bone formation or inhibit osteoclastmediated bone resorption has very important practical significance for the treatment of osteoporosis [9].

At present, among the many drugs used for the treatment of osteoporosis, parathyroid hormone is the only one used to promote bone formation and that is approved by the US Food and Drug Administration (FDA) [10]. However, the application of parathyroid hormone can lead to osteosarcoma, which limits its large-scale clinical application [11-13]. Thus, researchers have begun working to identify natural compounds that may promote bone formation [14]. Curculigoside is a natural phenolic glycoside compound produced by Curculigo orchioides Gaertn [15]. The pharmacological effects of curculigoside are quite extensive and include antioxidative, anti-inflammatory, antidepressant, and antiosteoporotic effects [16].

Adipose-derived stem cells (ADSCs) exhibit high potential to differentiate into multilineage cells, including osteoblasts, chondrocytes, adipocytes, and fibroblasts [17-19]. A theoretical inverse relationship exists between the osteogenic and adipogenic differentiation of ADSCs [20]. The specific inhibition of ADSC adipogenesis and a concomitant enhancement of ADSC osteogenesis may provide a novel therapeutic approach for the treatment of osteoporosis [21].

The PI3K/Akt signal transduction pathway is the downstream signal transduction pathway mediated by the PI3K family, and this pathway plays an important role in regulating cell differentiation [22, 23]. Akt is a downstream target of PI3K and is also a critical mediator of survival signals for the promotion of cell differentiation [24]. The PI3K-Akt signaling pathway regulates many normal cellular processes, and aberrant activation of the PI3K-Akt pathway has been widely implicated in osteogenic differentiation [25]. However, whether curculigoside plays a beneficial role in promoting the osteogenic differentiation of ADSCs is unknown.

In this study, we assessed the role of curculigoside in promoting the osteogenic differentiation of ADSCs, as well as the underlying mechanism, through in vivo and in vitro studies.

\section{Methods \\ Chemicals and reagents}

Curculigoside (chemical purity $=99.73 \%$ ) was purchased from MCE company (CAS: 85643-19-2, cat: HY-N0705, MedChemExpress, NJ, USA). Dulbecco's modified Eagle's medium (DMEM) and fetal bovine serum (FBS) were purchased from Gibco (Life Technologies, Shanghai, China). The ALP activity kit and ALP staining kit were purchased from Beyotime Company (Shanghai, China). Alizarin Red $\mathrm{S}$ was purchased from Solarbio Company (Beijing, China).

\section{ADSC isolation and culture}

Human subcutaneous adipose tissue was obtained using liposuction. The adipose tissue was cut into small pieces and digested with $0.075 \%$ type I collagenase and trypsin in a shaking water bath at $37{ }^{\circ} \mathrm{C}$ for $30 \mathrm{~min}$. Then, LDMEM culture medium containing $10 \%$ FBS was added and filtered with a 100- $\mu \mathrm{m}$ cell sieve to remove the undigested tissue mass. The cell suspension was centrifuged at $1500 \mathrm{rpm}$ to remove the supernatant and resuspended in $5 \mathrm{~mL}$ of DMEM $+20 \%$ FBS. When the cultured primary cells reached $70-80 \%$ confluence, they were subcultured and used for further studies. Cells at passages three to five were used for experiments.

\section{CCK-8 assays}

ADSCs $\left(1 \times 10^{3}\right.$ per well $)$ were plated in a 96-well plate and then treated with basal medium or basal medium containing curculigoside at concentrations of $1,2.5,5$, 10 , and $20 \mu \mathrm{mol} / \mathrm{L}$. After 1, 3, and 7 days, cell proliferation was determined by the CCK- 8 assay (Solarbio, Beijing, China) according to the manufacturer's instructions. After washing with $1 \mathrm{x}$ PBS, $10 \mu \mathrm{L}$ CCK-8 reagent plus $100 \mu \mathrm{L}$ basal DMEM medium was added per well and incubated at $37{ }^{\circ} \mathrm{C}$ for $2 \mathrm{~h}$. The absorbance at 450 $\mathrm{nm}$ was measured with a microplate reader (Multiskan FC, Thermo Scientific, Shanghai, China). The optical density (OD) values were measured, and the cell proliferation rate was calculated. 


\section{Osteogenic differentiation and cell treatments}

For osteogenic differentiation, ADSCs were incubated at $1 \times 10^{4}$ cells $/ \mathrm{cm}^{2}(2 \mathrm{~mL} /$ well $)$ in a 6 -well plate. When the cell confluence reached $80 \%$, the ADSCs were treated with osteogenic induction medium containing $0,1,2.5$, and $5 \mu \mathrm{mol} / \mathrm{L}$ curculigoside. Osteogenic induction medium mainly included DMEM supplemented with 10 nM dexamethasone, 50 mug ml-1 ascorbic acid, and 0.1 $\mathrm{mM}$ beta-glycerophosphate.

\section{ALP activity determination}

Intracellular ALP activity was measured using a colorimetric ALP assay (Sigma Aldrich) as described previously at all four indicated timepoints and under all indicated conditions. Then, the absorbance of the solution at $450 \mathrm{~nm}$ was measured using a microplate reader (Multiskan FC, Thermo Fisher Scientific, Shanghai, China).The results for ALP activity were normalized to the level of the total protein as determined by the Bradford method.

\section{Alizarin Red S staining}

After 21 days, the cells were fixed in $4 \%$ paraformaldehyde at room temperature for $30 \mathrm{~min}$. Then, ADSCs were washed twice with PBS and stained with Alizarin Red S (0.2\%, pH 8.3) for $30 \mathrm{~min}$ at room temperature. After washing with distilled deionized water, the ADSCs were observed and photographed under an optical microscope (Leica DM 500, Leica light microscope, Wetzlar, Germany). To quantify mineralization, calcium deposits were desorbed using 10\% cetylpyridinium chloride (Sigma-Aldrich; Merck KGaA), and the absorbance at $570 \mathrm{~nm}$ was measured in triplicate plates using a microtiter plate reader.

\section{Real-time quantitative PCR}

Total RNA was extracted from ADSCs using TRIzol (Invitrogen) following the manufacturer's protocol. cDNA was synthesized using the cDNA Reverse Transcription kit (TAKARA) according to the manufacturer's instructions. RT-qPCR analyses were conducted by using SYBR Green PCR master mix (Takara, Tokyo, Japan) in the Light Cycler 480 Real-time PCR system (Roche Molecular Systems, Basel, Switzerland). The multiple change was calculated by $2^{-\Delta \Delta C t}$ method.

\section{Western blotting assays}

After the indicated treatment, ADSCs were washed twice with prechilled PBS buffer. The harvested ADSCs were lysed on ice for $30 \mathrm{~min}$ in RIPA lysis buffer (Beyotime Biotechnology, Shanghai, China) containing $100 \mathrm{mM}$ PMSF (Beyotime Biotechnology) to extract total proteins. The protein concentrations were measured by the BCA protein assay kit (Solarbio, Beijing, China). Then, equal amounts of protein extracts $(25 \mu \mathrm{g})$ were subjected to $10 \%$ SDS-PAGE as described. Next, the proteins were transferred electrophoretically to polyvinylidene difluoride (PVDF) membranes (Millipore Corporation, Billerica, MA, USA).

After blocking with 5\% skim milk for $2 \mathrm{~h}$ at room temperature, the membranes were incubated with specific primary antibodies against RUNX2, ALP, and Osterix (Santa Cruz, CA, USA) overnight at $4{ }^{\circ} \mathrm{C}$. The membranes were then washed three times with TBSTween 20 and incubated with HRP-conjugated secondary antibodies for $1 \mathrm{~h}$ at room temperature. Immunoreactive bands were visualized with ECL plus (GE Life Sciences) according to the manufacturer's instructions. The protein band densities were analyzed using Image software (version 18.0, National Institutes of Health, Bethesda, MD, USA).

\section{OVX-induced osteoporosis mouse model establishment and curculigoside treatment}

All the experiments were performed according to protocols approved by the Institutional Animal Care Committee of The First People's Hospital of Lianyungang. Eightweek-old female C57BL/6 mice were purchased from the Beijing Animal Institute (Beijing, China) and randomly divided into four groups (Sham group, OVX group, OVX + Curculigoside group, and OVX + Curculigoside +LY294002 group, $n=8$ ). Curculigoside was dissolved in dimethylsulfoxide (DMSO) and diluted in PBS.

Bilateral ovariectomy was performed to establish an OVX mouse model of osteoporosis. In the sham procedure, animals were anesthetized, and the ovaries were not removed. For the OVX + curculigoside group, curculigoside was administered via an intraperitoneal injection of $100 \mu \mathrm{l}$ curculigoside $(7.5 \mathrm{mg} / \mathrm{kg}$ body weight) for 4 weeks. For LY294002 administration, the mice were intraperitoneally injected with LY294002 (4 5 g per gram body weight) 1 week after curculigoside treatment.

\section{Histological and immunohistochemical analyses}

After fixation of the distal femur in 4\% paraformaldehyde, the femur was decalcified in $0.5 \mathrm{M}$ EDTA in PBS for 2 months. The EDTA solution was replaced every day until complete decalcification had occurred. The samples were then paraffin-embedded, and $5-\mu \mathrm{m}$ sections were produced. Then, HE staining was performed with a HE staining kit (Beyotime, China) according to the manufacturer's instructions.

The paraffin sections were incubated for $1 \mathrm{~h}$ at $70{ }^{\circ} \mathrm{C}$, deparaffinized in xylene, and rehydrated in graded ethanol. Then, endogenous peroxidase activity was quenched by $3 \% \mathrm{H}_{2} \mathrm{O}_{2}$ for $10 \mathrm{~min}$. The sections were deparaffinized, and antigen retrieval was performed by boiling for 
15 min in a microwave oven in $10 \mathrm{mM}$ citric acid (pH 6.0). The sections were blocked with 5\% BSA (Solarbio; Beijing, China) at room temperature for $60 \mathrm{~min}$ and incubated with primary anti-RUNX2 antibody and p-Akt antibody at room temperature for $2 \mathrm{~h}$. After washing three times in PBS, the sections were incubated with biotinylated anti-rabbit secondary antibodies (Boster, China) in PBS for $30 \mathrm{~min}$ at $37{ }^{\circ} \mathrm{C}$, incubated with avidin-biotin-peroxidase solution (SABC kit, Boster, China), and visualized with a DAB kit (Boster, China). Immunoreactivity was visualized with a 3,3'-diaminobenzidine tetrahydrochloride kit (ZSGB-BIO, Beijing, China).

\section{Statistical analyses}

Each group was analyzed in triplicate. We conducted statistical analysis using SPSS 20.0 (SPSS, Chicago, IL). The data are presented as the mean \pm SD. Differences among multiple groups were analyzed using one-way analysis of variance (ANOVA) followed by Dunnett's multiple comparison post hoc test. $P<0.05$ was considered statistically significant.

\section{Results}

\section{Curculigoside effectively promotes the proliferation of} ADSCs

The CCK- 8 results indicated that there were no significant differences between the groups treated different concentrations of curculigoside for $24 \mathrm{~h}$ (Fig. 1a). The CCK-8 assay indicated that curculigoside did not induce cytotoxicity at a concentration of $5 \mu \mathrm{mol} / \mathrm{L}$ at $48 \mathrm{~h}$ (Fig. 1b). The CCK- 8 results at $72 \mathrm{~h}$ were consistent with the CCK-8 result at $48 \mathrm{~h}$ (Fig. 1c). We therefore chose 5 $\mu \mathrm{mol} / \mathrm{L}$ curculigoside as an optimal dose for further investigations.

\section{Bioinformatic analysis of curculigoside}

To demonstrate the function of curculigoside, we constructed a compound-gene interaction network using the STITCH database (http://stitch.embl.de/). The analysis of the data from the STITCH database indicated that VEGFB and VEGFA were the target genes of curculigoside (Fig. 2a).

The biological process of target genes of curculigoside is displayed in Fig. 2b, which indicates that the target genes of curculigoside were mainly enriched in positive regulation of epithelial cell proliferation, transmembrane receptor protein tyrosine kinase signaling pathway, positive regulation of cell migration, positive regulation of endothelial cell proliferation, positive regulation of epithelial cell migration, positive regulation of MAPK cascade, positive regulation of phosphorylation, vascular endothelial growth factor receptor signaling pathway, positive regulation of multicellular organismal process, and vascular endothelial growth factor signaling pathway.

The cellular components of the target genes of curculigoside are shown in Fig. 2c, which indicates that the target genes of curculigoside were mainly enriched in vesicle lumen, platelet alpha granule lumen, platelet alpha granule, extracellular space, cytoplasmic vesicle part, cytoplasmic vesicle, sorting endosome, cytoplasmic membrane-bounded vesicle, extracellular region, and extracellular region part.

The molecular functions of the target genes of curculigoside are shown in Fig. 2d, which indicates that the target genes of curculigoside were mainly enriched in vascular endothelial growth factoractivated receptor activity, receptor binding, transmembrane receptor protein tyrosine kinase activity, protein binding, protein tyrosine kinase activity, protein complex binding, integrin binding, vascular endothelial growth factor receptor 1 binding, growth factor binding, and carbohydrate derivative binding.
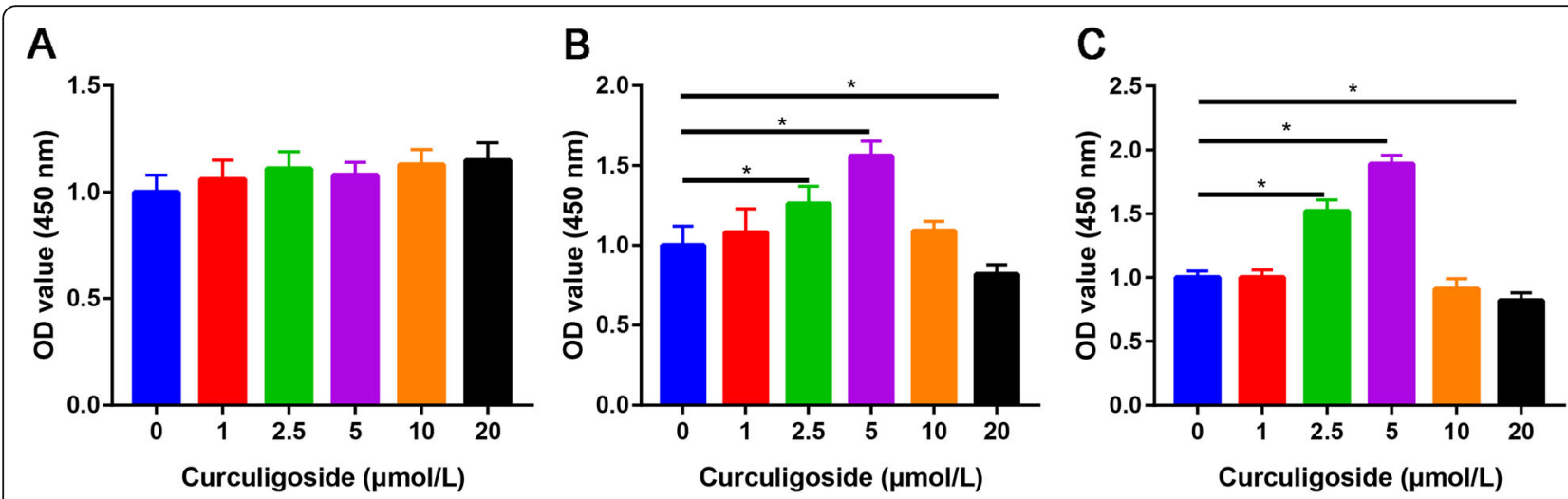

Fig. 1 The effects of curculigoside on ADSC growth at 24, 48, and $72 \mathrm{~h}$ were assessed by CCK-8 assay. CCK-8 assay of cell proliferation in ADSCs after treatment with different concentrations of curculigoside at $24 \mathrm{~h}(\mathbf{a}), 48 \mathrm{~h}(\mathbf{b})$, and $72 \mathrm{~h}(\mathbf{c})$ 


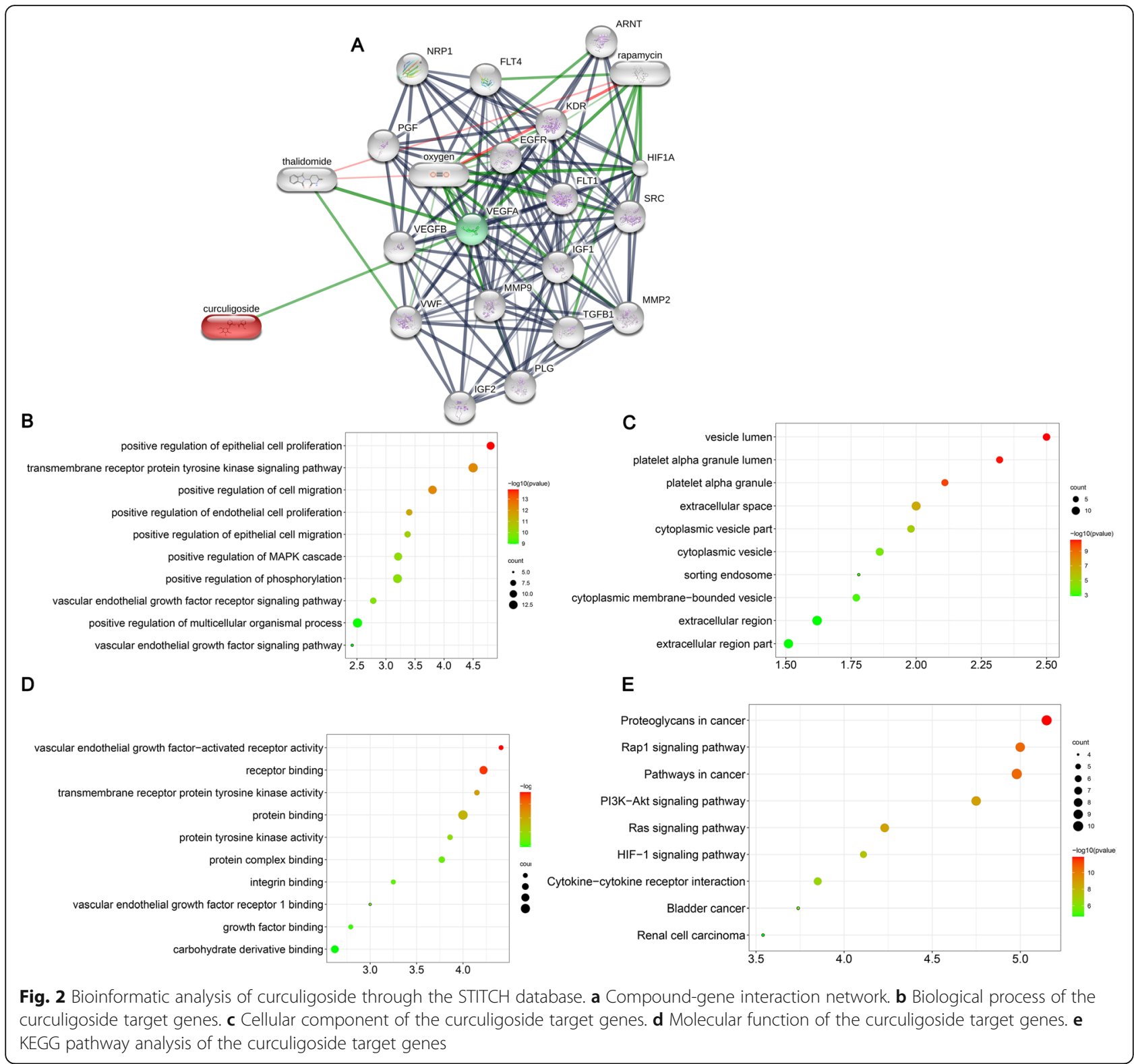

KEGG enrichment analysis indicated that the target genes of curculigoside were mainly involved in proteoglycans in cancer, Rap1 signaling pathway, pathways in cancer, PI3K-Akt signaling pathway, Ras signaling pathway, HIF-1 signaling pathway, cytokine-cytokine receptor interaction, bladder cancer, and renal cell carcinoma (Fig. 2e).

\section{Curculigoside effectively promotes osteogenic differentiation of ADSCs}

To explore the function of curculigoside on the osteogenic differentiation of ADSCs, we performed an ALP activity assay and AR staining. The ALP and ARS results revealed that the induced group had higher ALP activity and calcium deposition than the control group.
Moreover, curculigoside promoted biomineralization along with ALP activity and ARS staining compared to the induced and control groups, and these effects were partially inhibited by LY294002 (Fig. 3).

\section{Curculigoside effectively promotes osteogenic differentiation of ADSCs}

The RT-PCR results indicated that osteogenic induction significantly increased RUNX2, ALP, and Osterix expression (Fig. 4a). The curculigoside group also exhibited significantly increased RUNX2, ALP, and Osterix expression compared with the osteogenic induction group (Fig. $4 a)$, whereas these promotion effects were partially reversed by the PI3K inhibitor LY294002 (Fig. 4a). The western blot analysis results were consistent with the 

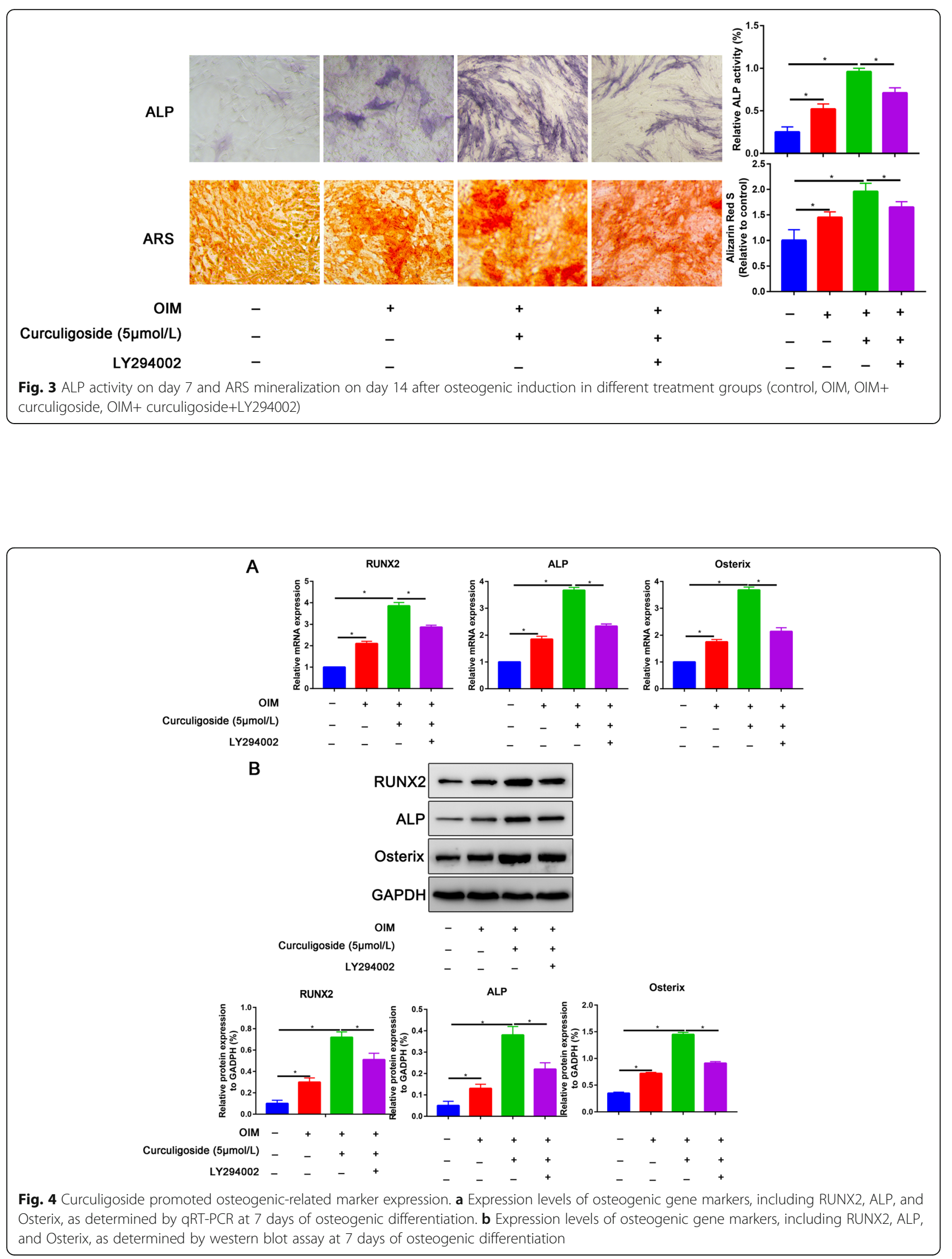
qRT-PCR results, showing that the protein expression of RUNX2, ALP, and Osterix was upregulated in curculigoside-treated ADSCs but was partially reversed by the PI3K inhibitor LY294002 (Fig. 4b).

\section{PI3K/Akt signaling is activated by curculigoside-treated ADSCs}

The phosphorylation of AKT to P-AKT is central to the PI3K/Akt pathway, and its activation stimulates the osteogenic differentiation of ADSCs. As shown in Fig. 5, phosphorylation of AKT to P-AKT was increased after osteogenic induction of ADSCs. The curculigoside group also exhibited significantly increased phosphorylation of AKT to P-AKT compared with the osteogenic induction group (Fig. 5), whereas the phosphorylation of AKT to P-AKT was partially reversed by the PI3K inhibitor LY294002.

\section{Curculigoside prevented bone mass loss in the OVX mouse model}

HE staining showed that the trabecular bone was sparse and reduced in the OVX model group compared to the sham group. However, after treatment with curculigoside, the mice had a higher bone volume than the OVX mice, suggesting partial protection from cancellous bone loss. In addition, when LY294002 was preadded, the protective effects of curculigoside could be neutralized (Fig. 6).

Immunofluorescence staining found significantly downregulated expression of RUNX2 in the OVX mice compared with the sham mice. However, after treatment with curculigoside, the mice had a higher expression of RUNX2, suggesting that curculigoside could enhance RUNX2 expression and protect against OVX-induced bone loss (Fig. 6).
Additionally, p-AKT expression in osteoblasts was lower in OVX mice, while after treatment with curculigoside, p-Akt expression was partially elevated. In addition, when LY294002 was added, p-Akt expression was partially neutralized (Fig. 6).

\section{Discussion}

In this study, we found that curculigoside did not induce cytotoxicity at a concentration of $5 \mu \mathrm{mol} / \mathrm{L}$ at $48 \mathrm{~h}$. We then verified that curculigoside can promote the osteogenic differentiation of ADSCs through the PI3K/Akt signaling pathway in vitro and in vivo.

In this study, we first identified the optimal dose of curculigoside for ADSC proliferation. We measured ADSC viability for up to $72 \mathrm{~h}$ and found that the optimal dose of curculigoside was $5 \mu \mathrm{mol} / \mathrm{L}$. Then, ALP and ARS staining were performed to assess the osteogenic effect of curculigoside on ADSCs. The results suggested that curculigoside plays a positive role in promoting the osteogenic differentiation of ADSCs. To further explore the mechanism underlying the effect of curculigoside in the osteogenic differentiation of ADSCs, the mechanism associated with curculigoside was further analyzed using the STITCH database to explore chemical-protein interactions. The STITCH database was utilized for the construction of compound-target and compound-target pathways. PI3K/Akt was the most significantly enriched pathway. Zhang et al. [26] found that curculigoside protects against excess iron-induced bone loss by attenuating Akt-FoxO1-dependent oxidative damage in mice and osteoblastic MC3T3-E1 cells. Curculigoside, one of the main bioactive phenolic compounds isolated from the rhizome of Curculigo orchioides Gaertn, is reported to have potent antioxidant and anti-osteoporotic properties. However, whether curculigoside plays a beneficial

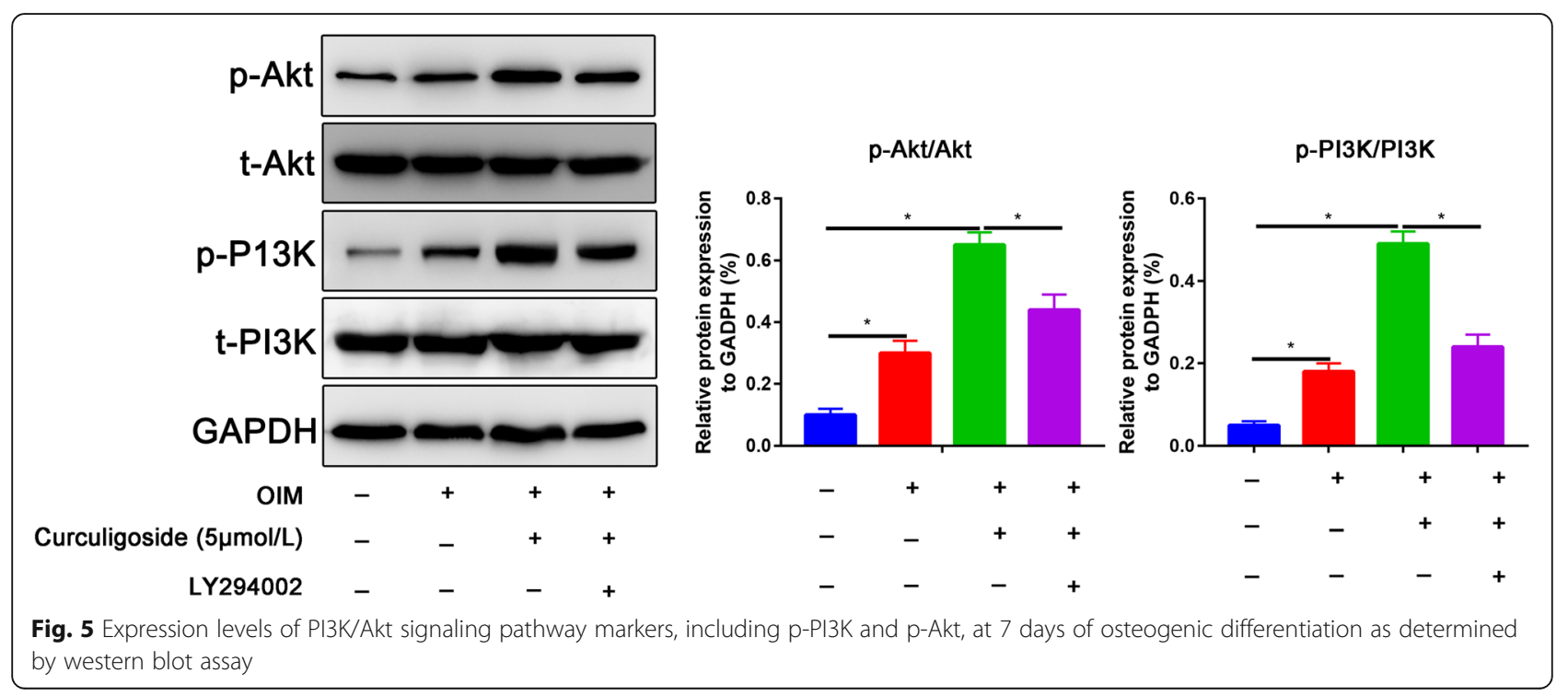




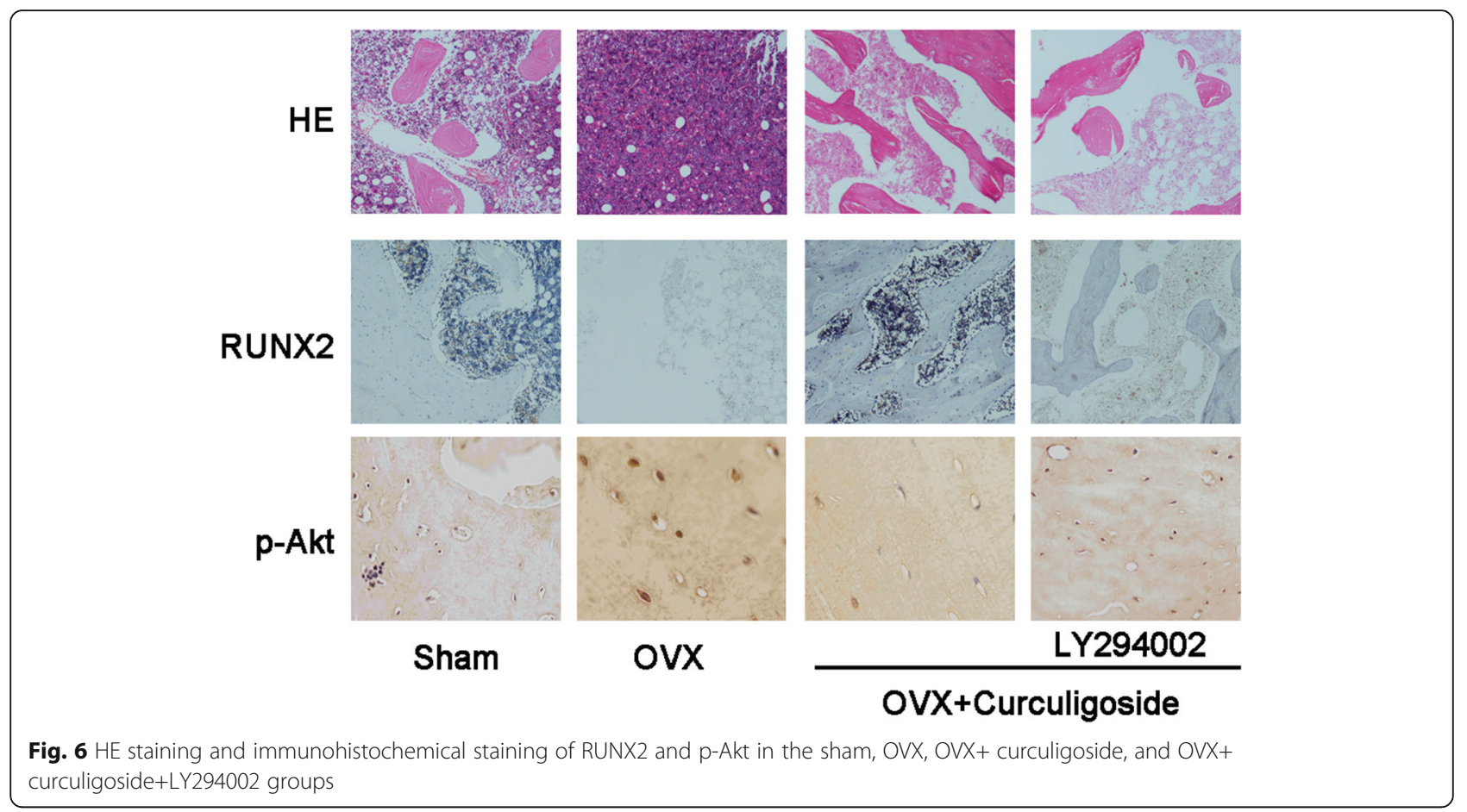

role in promoting osteogenic differentiation of ADSCs is unknown.

The PI3K-AKT signaling pathway is activated in the osteogenic differentiation of multiple stem cells, including ADSCs [27]. The PI3K/AKT signaling pathway has been shown to be critical for all phases of osteoblast differentiation and maturation, bone development, and growth [28]. Blocking the PI3K/AKT signaling pathway has also been shown to impair longitudinal bone growth and to prevent osteoblast differentiation [29]. In this study, we also found that p-PI3K and p-AKT expression was increased in ADSCs undergoing osteogenic induction and in curculigoside-treated ADSCs. Blocking the PI3K/Akt signaling pathway partially reversed the promoting effects of curculigoside on the osteogenic differentiation of ADSCs. Han et al. [15] predicted targets of curculigoside A in osteoporosis and rheumatoid arthritis using network pharmacology and experimental verification. The results showed that curculigoside A mainly targeted the EGFR, MAP2K1, MMP2, FGFR1, and MCL1 genes. These genes were involved in one critical signaling pathway, namely, the PI3K/Akt pathway.

Our study has numerous limitations. First, which receptor or protein is associated with the effect of curculigoside in ADSCs is unknown, and thus, more studies are needed. Second, the use of animal models seems to be necessary to evaluate the safety of curculigoside in vivo. The optimal dose of curculigoside for the treatment of osteoporosis in a large animal model is unknown.
Hence, the most suitable dose needs to be verified by further prospective large-scale studies.

\section{In conclusion}

Our data indicated that curculigoside induced the osteogenic differentiation and mineralization of ADSCs by activating the PI3K/Akt pathway in vitro and protected against OVX-induced bone loss in vivo. Curculigoside may be a potential candidate for the pharmacological treatment of osteoporosis.

\section{Abbreviations}

ALP: Alkaline phosphatase; ARS: Alizarin red S; STITCH: Search tool for interactions of chemicals; ADSCs: Adipose-derived stem cells;

OVX: Ovariectomized; FDA: Food and Drug Administration; PBS: Phosphatebuffered saline; OD: Optical density; PVDF: Polyvinylidene difluoride;

ECL: Electrochemiluminescence; DMSO: Dimethylsulfoxide

\section{Acknowledgements}

Not applicable.

\section{Authors' contributions}

XMY, YQS, and LL designed the study and conducted the experiments. DML and GDC performed the statistically analyses. GDC and XMY wrote the draft. YQS and LL edited and approved the final manuscript. All the authors read and approved the final manuscript.

\section{Funding}

There is no funding for this article.

Availability of data and materials

All the data pertaining to the present study have been included in figure in the manuscript, and the authors are willing to share the raw data upon reasonable request. 


\section{Declarations}

Ethics approval and consent to participate

Not applicable.

\section{Consent for publication}

Not applicable.

\section{Competing interests}

The authors declare that they have no competing interests.

\section{Author details}

'Department of Pharmacy, The First People's Hospital of Lianyungang, No. 128, Tongguanbei Road, Haizhou District, Lianyungang 222002, Jiangsu Province, China. 'Department of Pharmacy, Xinghua City People's Hospital, Xinghua City, Jiangsu Province, China.

\section{Received: 1 March 2021 Accepted: 30 March 2021}

Published online: 26 April 2021

\section{References}

1. Zhao Z, Ma X, Ma J, Sun X, Li F, Lv J. Naringin enhances endothelial progenitor cell (EPC) proliferation and tube formation capacity through the CXCL12/CXCR4/PI3K/Akt signaling pathway. Chem Biol Interact. 2018;286: 45-51. https://doi.org/10.1016/j.cbi.2018.03.002.

2. Sadile F, Bernasconi A, Russo S, Maffulli N. Core decompression versus other joint preserving treatments for osteonecrosis of the femoral head: a metaanalysis. Br Med Bull. 2016;118(1):33-49. https://doi.org/10.1093/bmb/ldw010.

3. Chen YS, Kang XR, Zhou ZH, et al. MiR-1908/EXO1 and MiR-203a/FOS, regulated by $s c d 1$, are associated with fracture risk and bone health in postmenopausal diabetic women. Aging (Albany NY). 2020;12:9549-84. https://doi.org/10.18632/aging.103227

4. Song $N$, Zhao Z, Ma X, Sun X, Ma J, Li F, et al. Naringin promotes fracture healing through stimulation of angiogenesis by regulating the VEGFNEGFR2 signaling pathway in osteoporotic rats. Chem Biol Interact. 2017;261:11-7. https://doi.org/10.1016/j.cbi.2016.10.020.

5. Tao Z, Wang J, Wen K, Yao R, da W, Zhou S, et al. Pyroptosis in osteoblasts: a novel hypothesis underlying the pathogenesis of osteoporosis. Front Endocrinol (Lausanne). 2020;11:548812. https://doi.org/10.3389/fendo.2020. 548812.

6. Udagawa N, Koide M, Nakamura M, Nakamichi Y, Yamashita T, Uehara S, et al. Osteoclast differentiation by RANKL and OPG signaling pathways. J Bone Miner Metab. 2021;39(1):19-26. https://doi.org/10.1007/s00774-020-011 62-6.

7. Migliorini F, Maffulli N, Eschweiler J, Tingart M, Baroncini A. Core decompression isolated or combined with bone marrow-derived cell therapies for femoral head osteonecrosis. Expert Opin Biol Ther. 2021;21(3): 423-30. https://doi.org/10.1080/14712598.2021.1862790.

8. Borciani G, Montalbano G, Baldini N, Cerqueni G, Vitale-Brovarone C, Ciapetti G. Co-culture systems of osteoblasts and osteoclasts: Simulating in vitro bone remodeling in regenerative approaches. Acta Biomater. 2020;108:2245. https://doi.org/10.1016/j.actbio.2020.03.043

9. Shi $Y$, Shu H, Wang $X$, Zhao H, Lu C, Lu A, et al. Potential advantages of bioactive compounds extracted from traditional Chinese medicine to inhibit bone destructions in rheumatoid arthritis. Front Pharmacol. 2020;11:561962. https://doi.org/10.3389/fphar.2020.561962.

10. Mace ML, Olgaard K, Lewin E. New aspects of the kidney in the regulation of fibroblast growth factor 23 (FGF23) and mineral homeostasis. Int J Mol Sci. 2020;21(22). https://doi.org/10.3390/ijms21228810.

11. González-Parra E, Bover J, Herrero J, Sánchez E, Molina P, Martin-Malo A, et al. Control of phosphorus and prevention of fractures in the kidney patient. Nefrologia. 2021;41(1):7-14. https://doi.org/10.1016/j.nefro.2020.05.015.

12. Mediouni $M, D R S, M a d r y ~ H$, et al. A review of translational medicine. The future paradigm: how can we connect the orthopedic dots better? Curr Med Res Opin. 2018;34(7):1217-29. https://doi.org/10.1080/03007995.2017.13 85450.

13. MM. A new generation of orthopaedic surgeons: "T-model". Current Orthopaedic Practice. 2019;30(1):444-5. https://doi.org/10.1152/ajpcell. 00211.2018
14. Funato N. New insights into cranial synchondrosis development: a mini review. Front Cell Dev Biol. 2020;8:706. https://doi.org/10.3389/fcell.2020. 00706.

15. Han J, Wan M, Ma Z. Prediction of targets of curculigoside A in osteoporosis and rheumatoid arthritis using network pharmacology and experimental verification. Drug Des Devel Ther. 2020;14:5235-50. https://doi.org/10.2147/ dddt.s282112.

16. Wang S, Liu W, Wang J, Bai X. Curculigoside inhibits ferroptosis in ulcerative colitis through the induction of GPX4. Life Sci. 2020;259:118356. https://doi. org/10.1016/j.lfs.2020.118356

17. Chen M, Zhou M, Fu Y, Li J, Wang Z. Effects of miR-672 on the angiogenesis of adipose-derived mesenchymal stem cells during bone regeneration. Stem Cell Res Ther. 2021;12(1):85. https://doi.org/10.1186/s13287-021-02154-7.

18. Kim H, Han SH. A novel $3 \mathrm{D}$ indirect co-culture system based on a collagen hydrogel scaffold for enhancing the osteogenesis of stem cells. J Mater Chem B. 2020;8(41):9481-91. https://doi.org/10.1039/d0tb01770a.

19. Zhou Z, Lu Y, Wang $Y$, du L, Zhang $Y$, Tao J. Let-7c regulates proliferation and osteodifferentiation of human adipose-derived mesenchymal stem cells under oxidative stress by targeting SCD-1. Am J Physiol Cell Physiol. 2019; 316(1):C57-c69. https://doi.org/10.1152/ajpcell.00211.2018.

20. An Y, Zhao J, Nie F, Wu Y, Xia Y, Li D. Parathyroid hormone (PTH) promotes ADSC osteogenesis by regulating SIK2 and Wnt4. Biochem Biophys Res Commun. 2019;516(2):551-7. https://doi.org/10.1016/j.bbrc.2019.06.084.

21. Yang XM, Song YQ, Li L, Liu DM, Chen GD. miR-1249-5p regulates the osteogenic differentiation of ADSCs by targeting PDX1. J Orthop Surg Res. 2021:16(1):10. https://doi.org/10.1186/s13018-020-02147-x.

22. Ma Y, Ran D, Zhao H, Song R, Zou H, Gu J, et al. Cadmium exposure triggers osteoporosis in duck via P2X7/PI3K/AKT-mediated osteoblast and osteoclast differentiation. Sci Total Environ. 2021;750:141638. https://doi.org/10.1016/j. scitotenv.2020.141638.

23. Li M, Luo R, Yang W, Zhou Z, Li C. miR-363-3p is activated by MYB and regulates osteoporosis pathogenesis via PTEN/PI3K/AKT signaling pathway. In Vitro Cell Dev Biol Anim. 2019;55(5):376-86. https://doi.org/10.1007/s1162 6-019-00344-5.

24. Xi JC, Zang HY, Guo LX, Xue HB, Liu XD, Bai YB, et al. The PI3K/AKT cell signaling pathway is involved in regulation of osteoporosis. J Recept Signal Transduct Res. 2015;35(6):640-5. https://doi.org/10.3109/10799893.2015.1041 647.

25. Xiao Y, Li B, Liu J. MicroRNA-148a inhibition protects against ovariectomyinduced osteoporosis through PI3K/AKT signaling by estrogen receptor a. Mol Med Rep. 2018;17:7789-96. https://doi.org/10.3892/mmr.2018.8845.

26. Zhang Q, Zhao L, Shen Y, He Y, Cheng G, Yin M, et al. Curculigoside protects against excess-iron-induced bone loss by attenuating Akt-FoxO1dependent oxidative damage to mice and osteoblastic MC3T3-E1 cells. Oxid Med Cell Longev. 2019;2019:9281481-14. https://doi.org/10.1155/2019/92 81481.

27. Zhang W, Bai X, Zhao B, Li Y, Zhang Y, Li Z, et al. Cell-free therapy based on adipose tissue stem cell-derived exosomes promotes wound healing via the PI3K/Akt signaling pathway. Exp Cell Res. 2018;370(2):333-42. https://doi. org/10.1016/j.yexcr.2018.06.035

28. Ye C, Zhang W, Hang K, Chen M, Hou W, Chen J, et al. Extracellular IL-37 promotes osteogenic differentiation of human bone marrow mesenchymal stem cells via activation of the PI3K/AKT signaling pathway. Cell Death Dis. 2019;10(10):753. https://doi.org/10.1038/s41419-019-1904-7.

29. Mukherjee A, Larson EA, Klein RF, Rotwein P. Distinct actions of akt1 on skeletal architecture and function. PLoS One. 2014;9(3):e93040. https://doi. org/10.1371/journal.pone.0093040.

\section{Publisher's Note}

Springer Nature remains neutral with regard to jurisdictional claims in published maps and institutional affiliations. 